\title{
Mycobacterium insubricum sp. nov.
}

\author{
Correspondence \\ Enrico Tortoli \\ e.tortoli@libero.it
}

\section{Enrico Tortoli, ${ }^{1}$ Serena Baruzzo, ${ }^{1}$ Yvonne Heijdra, ${ }^{2}$ Hans-Peter Klenk, ${ }^{3}$ Stefania Lauria, ${ }^{1}$ Alessandro Mariottini ${ }^{4}$ and Jakko van Ingen ${ }^{5}$}

\author{
${ }^{1}$ Mycobacteriology Reference Center, Microbiology Laboratory, Careggi Hospital, Florence, Italy \\ ${ }^{2}$ Department of Pulmonary Diseases, Radboud University Nijmegen Medical Center, Nijmegen, \\ The Netherlands \\ ${ }^{3}$ DSMZ - German Collection of Microorganisms and Cell Cultures, Braunschweig, Germany \\ ${ }^{4}$ Cytogenetics and Genetics Unit, Careggi Hospital, Florence, Italy \\ ${ }^{5}$ National Mycobacteria Reference Laboratory, National Institute of Public Health and the \\ Environment, Bilthoven, The Netherlands
}

\begin{abstract}
Five independent strains, isolated from clinical samples but probably not responsible for disease, revealed phenotypic and genotypic features that appeared to exclude their belonging to any of the recognized Mycobacterium species. The strains, which are non-pigmented rapid growers, presented a cell-wall lipid pattern resembling those of Mycobacterium brumae and Mycobacterium fallax. Sequencing of the 16S rRNA, hsp65 and rpoB genes and the 16S-23S rRNA gene internal transcribed spacer (ITS) revealed that the strains are clearly distinct from every other Mycobacterium species. While the $16 \mathrm{~S}$ rRNA and rpoB genes were characterized by a single sequevar, two sequevars were detected in $h s p 65$ and three in the ITS. The divergence shown in the latter region was striking, in which only two short regions (less than $150 \mathrm{bp}$ in all) were comparable with other mycobacteria, apart from Mycobacterium monacense and Mycobacterium gilvum. The PCR restriction analysis pattern of the novel strains also differs from any reported to date. The name Mycobacterium insubricum sp. nov. is proposed for the novel species; the type strain is $\mathrm{FI}-06250^{\top}\left(=\mathrm{DSM} 45132^{\top}=\mathrm{CIP} 109609^{\top}\right)$.
\end{abstract}

The continuous evolution of molecular techniques has led, over the last 15 years, to an extraordinary increase in the number of described bacterial species. The genus Mycobacterium now numbers more than 130 species. This massive proliferation of novel species is the subject of debate, with some people considering it inappropriate to describe novel species that present only minor differences from those already recognized or that are represented by a single isolate (Drancourt \& Raoult, 2005; Tortoli, 2003).

Abbreviations: ITS, internal transcribed spacer; MIC, minimal inhibitory concentration; PRA, PCR restriction analysis.

The GenBank/EMBL/DDBJ accession numbers for the sequences of the $16 \mathrm{~S}$ rRNA gene and ITS of strains $\mathrm{FI}-06250^{\top}$ and $\mathrm{Fl}-06215$ are EU605695 and EU605694; that for the 16S rRNA gene sequence of $\mathrm{FI}-05244$ is DQ142672; those for the hsp 65 gene sequences of strains $\mathrm{Fl}-06250^{\top}, \mathrm{FI}-06215$ and $\mathrm{Fl}-05244$ are EF584487-EF584489; those for the ITS sequences of Fl-05244 and NLA000701900 are EF584486 and EU624330; and those for rpoB gene sequences of FI-06250 ${ }^{\top}, M$. fallax DSM 44179 ${ }^{\top}, M$. brumae CIP $103465^{\top}$ and $M$. triviale IP14033001 are EU022519 and EU999023-EU999025.

Phylogenetic trees based on $h s p 65$ and rpoB gene sequences of selected mycobacterial species and the fatty acid profiles of strains $\mathrm{FI}$ $06250^{\top}, \mathrm{Fl}-06215$ and $\mathrm{Fl}-05244$ are available as supplementary material with the online version of this paper.
We report here on a homogeneous cluster of five independent strains that differ clearly, at both phenotypic and genotypic levels, from any previously reported mycobacterium. These strains therefore appear to fulfil even the most restrictive criteria suggested for the recognition of novel species.

The mycobacteria characterized here, strains FI-06250 ${ }^{\mathrm{T}}$, FI06215, FI-05244, FI-03111 and NLA000701900, which are not pigmented and form visible colonies in less than a week, were isolated from sputa of patients with various pulmonary problems (Table 1). For at least three of them, clinical significance seems unlikely: other causes of disease could not be excluded, and each patient yielded a single isolate (Griffith et al., 2007).

Cultural tests and several biochemical investigations, selected from those used more frequently for description of novel mycobacteria, were performed on the five strains according to standard procedures (Kent \& Kubica, 1985). Three different methods were used to investigate the lipid composition of the cell wall. Two-dimensional TLC of whole-organism acid methanolysates was conducted following the standard procedure of Minnikin et al. (1975). GLC determination of methyl-branched fatty acids, 
Table 1. Clinical and microbiological data for the five novel strains

I, Italy; NL, Netherlands; NA, not available.

\begin{tabular}{|c|c|c|c|c|c|c|c|c|c|c|}
\hline Strain & \multicolumn{2}{|r|}{ Isolation } & \multicolumn{3}{|c|}{ Microbiological data } & \multicolumn{5}{|c|}{ Patient data } \\
\hline FI-05244 & 2005 & Busto Arsizio (I) & $\begin{array}{l}\text { One induced } \\
\text { sputum, one } \\
\text { gastric aspirate }\end{array}$ & Negative & 2 & 62 & $\begin{array}{l}\text { Respiratory insuf- } \\
\text { ficiency, astenia }\end{array}$ & Lung cancer & No & Death \\
\hline FI- $06250^{\mathrm{T}}$ & 2006 & Varese (I) & $\begin{array}{l}\text { Two sputa, one } \\
\text { bronchial aspirate, } \\
\text { one pleural fluid }\end{array}$ & Negative & 1 (sputum) & 62 & Pleural effusion & $\begin{array}{l}\text { Chronic obstructive pul- } \\
\text { monary disease }\end{array}$ & No & Lost \\
\hline NLA000701900 & 2007 & Nijmegen (NL) & Three sputa & Positive (3) & 3 & 23 & Cough, dyspnoea & $\begin{array}{l}\text { Chronic obstructive pul- } \\
\text { monary disease, bronch- } \\
\text { iectasis, uncharacterized } \\
\text { immunological deficit }\end{array}$ & No & Stable \\
\hline
\end{tabular}


alcohols and mycolic acid cleavage products was performed using the Microbial Identification System software package (Sasser, 1990). Finally, HPLC of cell-wall mycolic acids was performed after esterification to bromophenacyl esters (Butler \& Kilburn, 1988). While all the strains characterized here were submitted to HPLC, only strains FI- $06250^{\mathrm{T}}$, FI-06215 and FI-05244 were analysed with TLC and GLC.

Minimal inhibitory concentrations (MIC) of drugs recommended for rapidly growing mycobacteria were determined for all five strains according to the reference method of the NCCLS (2003) (now the CLSI).

Double-strand sequencing was carried out using BigDye Terminator chemistry and an AB3730 DNA sequencer (Applied Biosystems) following the standard procedures on four species-markers of primary importance for taxonomic and phylogenetic analysis of mycobacteria (Adékambi et al., 2003; McNabb et al., 2004; Reischl et al., 1998; Roth et al., 1998). In addition to the $16 \mathrm{~S}$ rRNA gene, universally considered the 'gold standard' (Böttger, 1996), the hypervariable region of the gene encoding the $65 \mathrm{kDa}$ heat-shock protein ( $h s p 65)$ and the transcribed spacer interposed between the $16 \mathrm{~S}$ and the $23 \mathrm{~S}$ rRNA genes (ITS) were sequenced. A fragment of the $r p o B$ gene, recently proposed for phylogenetic analysis of rapidly growing mycobacteria and characterized by its very high discriminative power (Adékambi \& Drancourt, 2004), was also investigated since our strains showed high growth rates.

Phylogenetic analysis was conducted by including the sequences of the 25 genotypically most closely related type strains found in GenBank (sequences retrieved from GenBank BLAST). When not present among these 25, the sequences of strains of Mycobacterium brumae, Mycobacterium fallax and Mycobacterium triviale (the species most closely related phenotypically) were added. As the $r p o B$ sequences of the type strains of the latter three species were not present in GenBank, strains of each species (M. brumae CIP $103465^{\mathrm{T}}$, M. fallax DSM $44179^{\mathrm{T}}$ and $M$. triviale IP14033001) were investigated. After the sequences had been standardized and aligned with CLUSTAL $\mathrm{W}$, trees were constructed according to the neighbour-joining method (Saitou \& Nei, 1987) and evaluated by bootstrap analysis based on 1000 replicates using the MEGA software version 4 (Tamura et al., 2007). The trees were rooted using Mycobacterium tuberculosis H37Ra as the outgroup.

The strains, which were acid-fast, grew rapidly (visible colonies within 3-6 days) on Löwenstein-Jensen medium at temperatures of $30-37^{\circ} \mathrm{C}$, producing rough colonies which remained unpigmented after exposure to light; no growth was observed at $42{ }^{\circ} \mathrm{C}$. Growth was inhibited on media with p-nitrobenzoic acid and hydroxylamine added, while all the strains grew in the presence of thiophene carboxylic acid and isoniazid. Scanty growth was exhibited on MacConkey agar by two of the five strains. Almost all of the standard biochemical tests (niacin production, nitrate reduction, $68{ }^{\circ} \mathrm{C}$ catalase activity, semi-quantitative catalase activity, 3-day arylsulfatase activity, $\beta$-glucosidase activity, urease activity and Tween 80 hydrolysis) were negative; only tellurite reduction was scored as positive.

TLC revealed the presence of only $\alpha$-mycolates; within the genus Mycobacterium, very few species (M. brumae, $M$. fallax and $M$. triviale) are characterized by this rare mycolic acid pattern.

The GLC profile was characterized by hexadecanoic acid and 9-methyl octadecenoic acids as major compounds and by the absence of alcohols; the full profile is given in Supplementary Table S1, available in IJSEM Online. On the basis of the acid composition obtained by GLC analysis, the pattern of $M$. brumae CIP $103465^{\mathrm{T}}$ bore the closest resemblance to those of the novel strains. High similarity was also observed for M. fallax DSM $44179^{\mathrm{T}}$ and M. triviale IP14033001, with only quantitative but not qualitative differences between the aforesaid species and our strains.

Identical HPLC patterns, characterized by the presence of a single narrow cluster of peaks eluting after 7 min (Fig. 1), were displayed by the five strains investigated. A thorough investigation of our HPLC mycobacterium library (available at http://www.mycobactoscana.it) revealed that only $M$. brumae, $M$. fallax and $M$. triviale present profiles that grossly resemble this pattern.

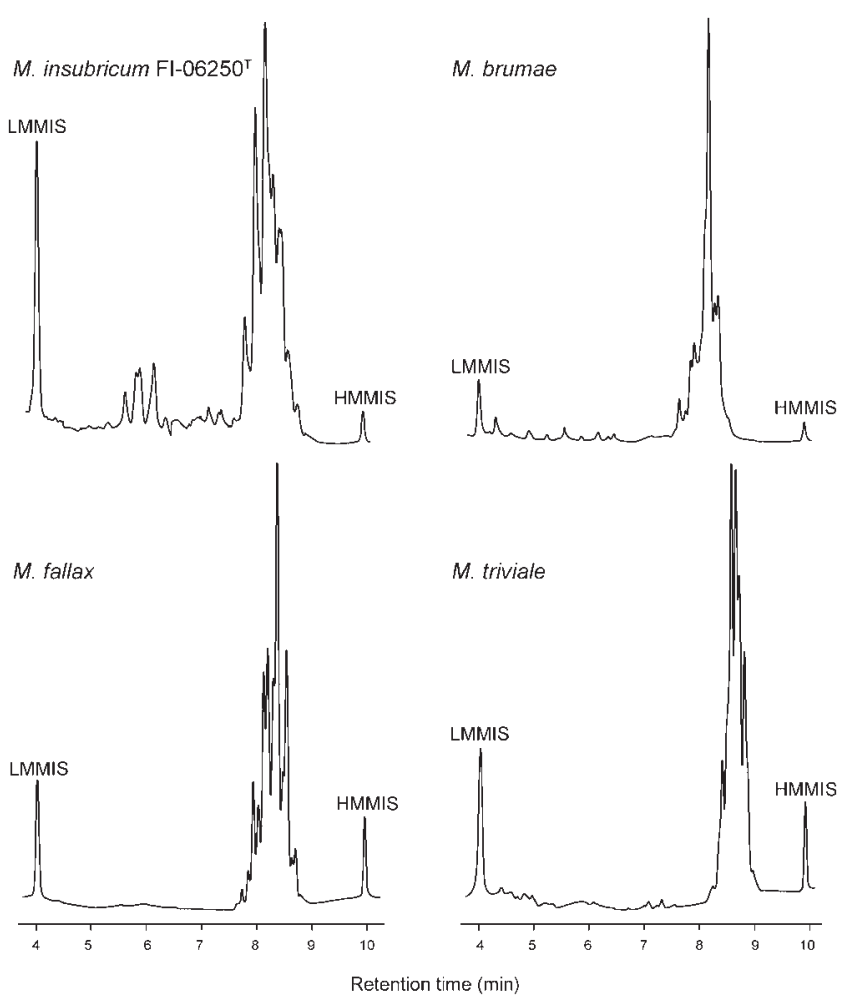

Fig. 1. HPLC patterns of mycolic acids of Mycobacterium insubricum sp. nov. $\mathrm{FI}-06250^{\top}, M$. brumae, $M$. fallax and $M$. triviale. LMMIS, Low-molecular-mass internal standard; HMMIS, high-molecular-mass internal standard. Patterns for reference species were taken from http://www.mycobactoscana.it. 
All the strains were characterized by low MICs for most of the antimicrobials tested, and resistance was not detected in any strain (Table 2).

The almost-complete 16S rRNA gene sequence was identical in the five strains and differed by 17 bp $(98.9 \%$ identity) from the type strain of Mycobacterium rhodesiae, which was the most closely related species. In the variable region of hsp65 (Telenti et al., 1993), two sequevars were detected that differed by a single cytosine/thymine substitution; the variants were present in two (FI-06215 and FI-05244) and three (the remaining strains) strains. The highest sequence identity was found with the type strains of M. fallax (13 mismatches in $401 \mathrm{bp;} 96 \%$ identity) followed by M. brumae, reflecting the relatedness that emerged at the level of cell-wall lipid composition.

The ITS was characterized by three sequevars: FI-03111 and FI-06215 presented two deletions, of 15 and 18 nucleotides, respectively, in comparison with FI-05244 and FI- $06250^{\mathrm{T}}$, while NLA000701900 differed from the latter two strains by a thymine deletion and a thymine/cytosine substitution (Fig. 2). Alignment of the ITS region was practically impossible because of the extensive divergence from other mycobacteria. Overall, the ITS sequences of the isolates had $70 \%$ identity with sequences from the type strains of Mycobacterium monacense and Mycobacterium gilvum; similarity with other mycobacteria was shown only over a shorter region. In the $r p o B$ gene, where the five strains shared a unique sequence, the type strains of $M$. brumae and $M$. fallax were the closest relatives, although with relatively low similarity (51 and 60 mismatches; identity of 92 and $90 \%$, respectively).

Table 2. MICs of the five novel strains

I, Intermediately susceptible; s, susceptible; -, no interpretation possible.

\begin{tabular}{|lcc|}
\hline Drug & MIC range $\left(\boldsymbol{\mu} \mathbf{~ m l}^{-\mathbf{1}}\right)$ & Interpretation $(\boldsymbol{n})$ \\
\hline Amikacin & $\leqslant 1$ & $\mathrm{~S}$ \\
Azlocillin/CA & $2-4$ & - \\
Cefoxitin & $4-16$ & $\mathrm{~S}$ \\
Ceftriaxone & $16-64$ & - \\
Ciprofloxacin & $\leqslant 0.12-0.25$ & $\mathrm{~S}$ \\
Clarithromycin & $\leqslant 0.12$ & $\mathrm{~S}$ \\
Ethambutol & $\leqslant 0.5-1$ & $\mathrm{~S}$ \\
Gatifloxacin & $\leqslant 0.06$ & $\mathrm{~S}$ \\
Imipenem & $2-8$ & $\mathrm{~S}(4) / \mathrm{I}(1)$ \\
Linezolid & $\leqslant 1$ & $\mathrm{~S}$ \\
Minocycline & $\leqslant 0.5$ & $\mathrm{~S}$ \\
Moxifloxacin & $\leqslant 0.06-0.5$ & $\mathrm{~S}$ \\
Rifabutin & $\leqslant 0.06-0.5$ & $\mathrm{~S}$ \\
Rifampicin & $\leqslant 0.06-4$ & $\mathrm{~S}(3) / \mathrm{I}(2)$ \\
Streptomycin & $1-4$ & $\mathrm{~S}(3) / \mathrm{I}(2)$ \\
Sulfamethoxazole & $\leqslant 5-10$ & $\mathrm{~S}$ \\
Tobramycin & $\leqslant 0.5$ & $\mathrm{~S}$ \\
\hline
\end{tabular}

420

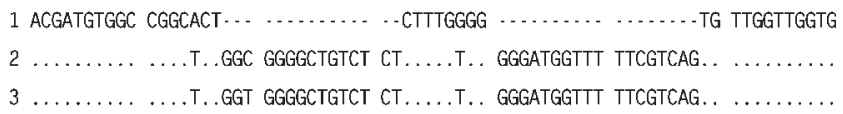

Fig. 2. Divergence in the sequence of the ITS region between strains $\mathrm{Fl}-03111$ (sequevar 1), $\mathrm{Fl}-06250^{\top}$ (sequevar 2) and NLA000701900 (sequevar 3).

For all four genetic regions investigated in this study, each strain differed from any other for at least one sequence.

PCR restriction analysis (PRA) inferred on the basis of restriction sites present in the $h s p 65$ sequences revealed a single pattern (despite the presence of two sequevars). BstEII produced theoretical fragments of 224, 106 and $94 \mathrm{bp}$, while the major theoretical products of HaeIII were 132, 87 and 58 bp long. No similar pattern was detected in the PRASITE database (http://app.chuv.ch/prasite/).

The phylogenetic tree constructed on the basis of the longest 16S rRNA gene stretch available in GenBank for all the species selected for the alignment (corresponding to positions 108-1467 of the Escherichia coli 16S rRNA gene sequence) showed Mycobacterium farcinogenes, Mycobacterium houstonense and Mycobacterium senegalense to be the most closely related species (Fig. 3). A similar approach was applied to a $401 \mathrm{bp}$ overlapping region within the hsp65 sequence (starting at position 442 of the hsp65 gene sequence of M. tuberculosis strain Erdman; GenBank accession no. M15467) (Takewaki et al., 1994). In this case, the species characterized by the highest relatedness to the test strains was M. fallax (Supplementary Fig. $\mathrm{S} 1$ ). In the analysis of a $723 \mathrm{bp}$ fragement of $r p o B$ (starting at position 2593 of the sequence of Mycobacterium smegmatis ATCC 14468; GenBank accession no. U24494), the closest species were $M$. brumae and $M$. fallax (Supplementary Fig. S2). Our attempts to analyse the ITS region did not produce significant alignments because of the unique characteristics of this genetic region in the novel strains mentioned above.

On the basis of the data presented here, we propose the name Mycobacterium insubricum sp. nov. to accommodate strains FI-06250 ${ }^{\mathrm{T}}$, FI-06215, FI-05244, FI-03111 and NLA000701900.

\section{Description of Mycobacterium insubricum sp. nov.}

Mycobacterium insubricum (in.su'bri.cum. L. neut. adj. insubricum from Insubria, the Latin name of part of the Lombardy region of Italy that includes the cities in which four of the first five strains were isolated, including the type strain).

Gram-positive-staining, acid-fast and non-motile. Does not form spores and produces smooth, white, nonchromogenic colonies within 7 days at $25-37{ }^{\circ} \mathrm{C}$. 


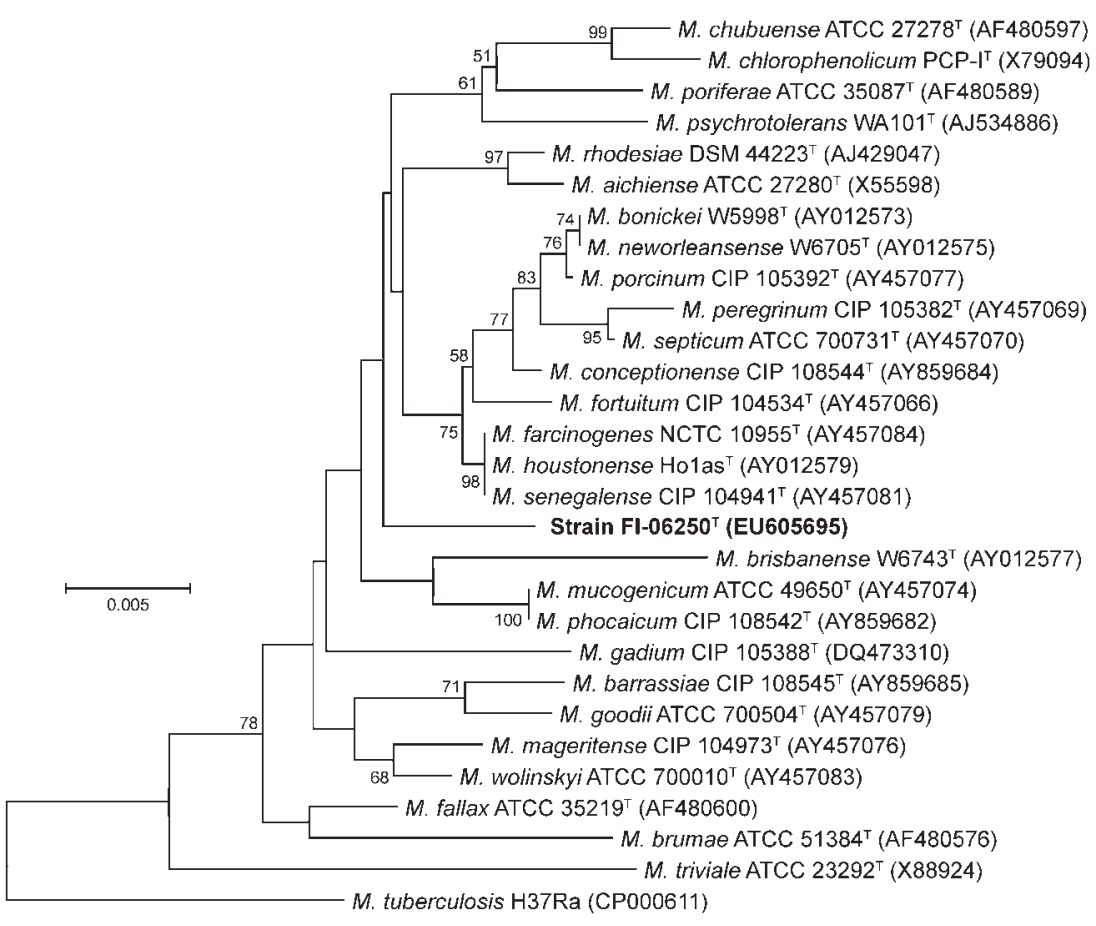

Fig. 3. Neighbour-joining phylogenetic tree based on $16 \mathrm{~S}$ rRNA gene sequences. $M$. tuberculosis H37Ra was used as the outgroup. Bootstrap values $\geqslant 50 \%$ are reported at nodes. Bar, $0.5 \%$ sequence difference.
Standard biochemical tests for identification of mycobacteria are negative with the exception of tellurite reduction and cannot be used for differentiation from other mycobacteria. The result of lipid investigations are noteworthy, with three approaches (TLC, GLC and HPLC) revealing a close resemblance to $M$. brumae, $M$. fallax and M. triviale. Susceptible in vitro to all of the most frequently used antimycobacterial drugs. Substantial divergence from other known mycobacteria is present in the sequences of the 16S rRNA, $h s p 65$ and $r p o B$ genes; observed divergence in these sequences generally exceeds the limits considered acceptable for differentiation of mycobacterial species (Turenne et al., 2001; Adékambi et al., 2003; Hall et al., 2003). The structure of the ITS shows low similarity to any other Mycobacterium species. The PRA pattern differs from any reported so far. The most closely related species on the basis of the phylogenetic analysis of the 16S rRNA gene sequence are $M$. farcinogenes, M. houstonense and $M$. senegalense.

The type strain, FI- $06250^{\mathrm{T}} \quad\left(=\mathrm{DSM} \quad 45132^{\mathrm{T}}=\mathrm{CIP}\right.$ $109609^{\mathrm{T}}$ ), was isolated from a sputum sample. Four other strains of the species, FI-06215, FI-05244, FI-03111 and NLA000701900, were isolated from sputum samples from independent patients with lung disease. It seems unlikely that the isolates were of clinical significance.

\section{References}

Adékambi, T. \& Drancourt, M. (2004). Dissection of phylogenetic relationships among 19 rapidly growing Mycobacterium species by $16 \mathrm{~S}$ rRNA, hsp65, sodA, recA and rpoB gene sequencing. Int J Syst Evol Microbiol 54, 2095-2105.
Adékambi, T., Colson, P. \& Drancourt, M. (2003). rpoB-based identification of nonpigmented and late-pigmenting rapidly growing mycobacteria. J Clin Microbiol 41, 5699-5708.

Böttger, E. C. (1996). Approaches for identification of microorganisms. Despite longer experience with fatty acid profiles, DNA-based analysis offers several advantages. ASM News 62, 247-250.

Butler, W. R. \& Kilburn, J. O. (1988). Identification of major slowly growing pathogenic mycobacteria and Mycobacterium gordonae by high-performance liquid chromatography of their mycolic acids. J Clin Microbiol 26, 50-53.

Drancourt, M. \& Raoult, D. (2005). Sequence-based identification of new bacteria: a proposition for creation of an orphan bacterium repository. J Clin Microbiol 43, 4311-4315.

Griffith, D. E., Aksamit, T., Brown-Elliott, B. A., Catanzarro, A., Daley, C., Gordin, F., Holland, S. M., Horsburg, R., Huitt, G. \& other authors (2007). An official ATS/IDSA statement: diagnosis, treatment, and prevention of nontuberculous mycobacterial diseases. Am J Respir Crit Care Med 175, 367-416.

Hall, L., Doerr, K. A., Wohlfiel, S. L. \& Roberts, G. D. (2003). Evaluation of the MicroSeq system for identification of mycobacteria by $16 \mathrm{~S}$ ribosomal DNA sequencing and its integration into a routine clinical mycobacteriology laboratory. J Clin Microbiol 41, 1447-1453.

Kent, P. T. \& Kubica, G. P. (1985). Public Health Mycobacteriology. A Guide for the Level III Laboratory. Atlanta, GA: US Department of Health and Human Services.

McNabb, A., Eisler, D., Adie, K., Amos, M., Rodrigues, M., Stephens, G., Black, W. A. \& Isaac-Renton, J. (2004). Assessment of partial sequencing of the 65-kilodalton heat shock protein gene (hsp65) for routine identification of Mycobacterium species isolated from clinical sources. J Clin Microbiol 42, 3000-3011.

Minnikin, D. E., Al-Shamaony, L. \& Goodfellow, M. (1975). Differentiation of Mycobacterium, Nocardia, and related taxa by thin-layer chromatographic analysis of whole-organism methanolysates. J Gen Microbiol 88, 200-204. 
NCCLS (2003). Susceptibility testing for mycobacteria, nocardiae and other aerobic actinomycetes. Approved standard M24-A. Wayne, PA: National Committee for Clinical Laboratory Standards.

Reischl, U., Emler, S., Horak, Z., Kaustova, J., Kroppenstedt, R. M., Lehn, N. \& Naumann, L. (1998). Mycobacterium bohemicum sp. nov., a new slow-growing scotochromogenic mycobacterium. Int $J$ Syst Bacteriol 48, 1349-1355.

Roth, A., Fisher, M., Hamid, M. E., Michalke, S., Ludwig, W. \& Mauch, H. (1998). Differentiation of phylogenetically related slowly growing mycobacteria based on 16S-23S rRNA gene internal transcribed spacer sequences. J Clin Microbiol 36, 139-147.

Saitou, N. \& Nei, M. (1987). The neighbor-joining method: a new method for reconstructing phylogenetic trees. Mol Biol Evol 4, 406-425.

Sasser, M. (1990). Identification of bacteria by gas chromatography of cellular fatty acids. MIDI Technical Note 101. Newark, DE: MIDI Inc.

Takewaki, S., Okuzumi, K., Manabe, I., Tanimura, M., Miyamura, K., Nakahara, K., Yazaki, Y., Ohkubo, A. \& Nagai, R. (1994). Nucleotide sequence comparison of the mycobacterial dnaJ gene and PCRrestriction fragment length polymorphism analysis for identification of mycobacterial species. Int J Syst Bacteriol 44, 159-166.

Tamura, K., Dudley, J., Nei, M. \& Kumar, S. (2007). MEGA 4: molecular evolutionary genetics analysis (MEGA) software version 4.0. Mol Biol Evol 24, 1596-1599.

Telenti, A., Marchesi, F., Balz, M., Bally, F., Böttger, E. C. \& Bodmer, T. (1993). Rapid identification of mycobacteria to the species level by polymerase chain reaction and restriction enzyme analysis. J Clin Microbiol 31, 175-178.

Tortoli, E. (2003). Impact of genotypic studies on mycobacterial taxonomy: the new mycobacteria of the 1990s. Clin Microbiol Rev 16, 319-354.

Turenne, C. Y., Tschetter, L., Wolfe, J. \& Kabani, A. (2001). Necessity of quality-controlled $16 \mathrm{~S}$ rRNA gene sequence databases: identifying nontuberculous Mycobacterium species. J Clin Microbiol 39, 36373648. 